\title{
A VARIANT OF A THEOREM BY AILON-RUDNICK FOR ELLIPTIC CURVES
}

\author{
D. GHIOCA, L.-C. HSIA, AND T. J. TUCKER
}

\begin{abstract}
Given a smooth projective curve $C$ defined over $\overline{\mathbb{Q}}$ and given two elliptic surfaces $\mathcal{E}_{1} \longrightarrow C$ and $\mathcal{E}_{2} \longrightarrow C$ along with sections $P_{i}, Q_{i}$ of $\mathcal{E}_{i}$ (for $i=1,2$ ), we prove that if there exist infinitely many $t \in C(\overline{\mathbb{Q}})$ such that for some integers $m_{1, t}, m_{2, t}$, we have that $\left[m_{i, t}\right]\left(P_{i}\right)_{t}=\left(Q_{i}\right)_{t}$ on $\mathcal{E}_{i}$ (for $i=1,2$ ), then at least one of the following conclusions must hold: either (i) there exists a nontrivial isogeny $\psi: \mathcal{E}_{1} \longrightarrow \mathcal{E}_{2}$ and also there exist nontrivial endomorphisms $\varphi_{i}$ of $\mathcal{E}_{i}$ (for $i=1,2$ ) such that $\varphi_{2}\left(P_{2}\right)=\psi\left(\varphi_{1}\left(P_{1}\right)\right)$; or (ii) $Q_{i}$ is a multiple of $P_{i}$ for some $i=1,2$. A special case of our result answers a conjecture made by Silverman.
\end{abstract}

\section{INTRODUCTION}

In [AR04], Ailon and Rudnick showed that for two multiplicatively independent nonconstant polynomials $a, b \in \mathbb{C}[x]$, there is a nonzero polynomial $h \in \mathbb{C}[x]$, depending on $a$ and $b$ such that $\operatorname{gcd}\left(a^{n}-1, b^{n}-1\right) \mid h$ for all positive integer $n$. In this paper, we prove a similar result for elliptic curves; instead of working with the multiplicative group $\mathbb{G}_{m}$, we work with the group law on an elliptic curve defined over a function field. The result of Ailon-Rudnick relies crucially on the Serre-Ihara-Tate theorem (see [Lan65]), while our result relies crucially on recent Bogomolov type results for elliptic surfaces due to DeMarco and Mavraki [DM].

Throughout our article, we work with elliptic surfaces over $\overline{\mathbb{Q}}$; more precisely, given a projective, smooth curve $C$ defined over $\overline{\mathbb{Q}}$, an elliptic surface $\mathcal{E} / C$ is given by a morphism $\pi: \mathcal{E} \longrightarrow C$ over $\overline{\mathbb{Q}}$ where the generic fiber of $\pi$ is an elliptic curve $E$ defined over $K=\overline{\mathbb{Q}}(C)$, while for all but finitely many $t \in C(\overline{\mathbb{Q}})$, the fiber $\mathcal{E}_{t}:=\pi^{-1}(\{t\})$ is an elliptic curve defined over $\overline{\mathbb{Q}}$. Recall that a section $\sigma$ of $\pi$ (i.e. a map $\sigma: C \longrightarrow \mathcal{E}$ such that $\pi \circ \sigma=\left.\mathrm{id}\right|_{C}$ ) gives rise to a $K$-rational point of $E$. Conversly, a point $P \in E(K)$ corresponds to a section of $\pi$; if we need to indicate the dependence with $P$, we will denote it by $\sigma_{P}$. So, for all but finitely many $t \in C(\overline{\mathbb{Q}})$, the intersection of the image of $\sigma_{P}$ in $\mathcal{E}$ with the fiber above $t$ is a point $P_{t}:=\sigma_{P}(t)$ on the elliptic curve $E_{t}$. For any integer $k$, the multiplication-by- $k$ map $[k]$ on $E$ extends

2010 AMS Subject Classification: Primary 11G50; Secondary 11G35, 14G25. The research of the first author was partially supported by an NSERC Discovery grant. The second author was supported by MOST grant 104-2115-M-003-004-MY2. The third author was partially supported by NSF Grant DMS-0101636. 
to a morphism on $\mathcal{E}$; if there is no danger of confusion, we still denote the extension by $[k]$.

We prove the following result.

Theorem 1.1. Let $\pi_{i}: \mathcal{E}_{i} \longrightarrow C$ be elliptic surfaces over a curve $C$ defined over $\overline{\mathbb{Q}}$ with generic fibers $E_{i}$, and let $\sigma_{P_{i}}, \sigma_{Q_{i}}$ be sections of $\pi_{i}$ (for $i=1,2$ ) corresponding to points $P_{i}, Q_{i} \in E_{i}(\overline{\mathbb{Q}}(C))$. If there exist infinitely many $t \in C(\overline{\mathbb{Q}})$ for which there exist some $m_{1, t}, m_{2, t} \in \mathbb{Z}$ such that $\left[m_{i, t}\right] \sigma_{P_{i}}(t)=$ $\sigma_{Q_{i}}(t)$ for $i=1,2$, then at least one of the following properties must hold:

(i) there exist isogenies $\varphi: E_{1} \longrightarrow E_{2}$ and $\psi: E_{2} \longrightarrow E_{2}$ such that $\varphi\left(P_{1}\right)=\psi\left(P_{2}\right)$.

(ii) for some $i \in\{1,2\}$, there exists $k_{i} \in \mathbb{Z}$ such that $\left[k_{i}\right] P_{i}=Q_{i}$ on $E_{i}$.

We note here that, in contrast to similar results such as [AR04] and [HT], the underlying multiplication morphisms need not be isotrivial (that is, they need not be defined over the field of constants in $k(C)$ ).

A special case of our result (when both $Q_{1}$ and $Q_{2}$ are the zero elements) answers in the affirmative [Sil04b, Conjecture 7]; this is carried out in a more general setting in our Proposition 4.3 from Section 4.

Silverman's question [Sil04b, Conjecture 7] was motivated by work of Ailon-Rudnick [AR04], who showed that the greatest common divisor of $a^{n}-1$ and of $b^{n}-1$ for multiplicatively independent polynomials $a, b \in \mathbb{C}[T]$ has bounded degree (see also the generalization by Corvaja-Zannier [CZ13b] along with the related results from [CZ08, CZ11, CZ13a]). In turn, the result of Ailon-Rudnick was motivated by the work of Bugeaud-Corvaja-Zannier [BCZ03] who obtained an upper bound for $\operatorname{gcd}\left(a^{k}-1, b^{k}-1\right)$ (as $k$ varies in $\mathbb{N}$ ) for given $a, b \in \overline{\mathbb{Q}}$. On the other hand, Silverman [Sil04a] showed that the degree of $\operatorname{gcd}\left(a^{m}-1, b^{n}-1\right)$ could be quite large when $a, b \in \overline{\mathbb{F}_{p}}[T]$; see also the authors' previous paper [GHT17] where we study the $\operatorname{gcd}\left(a^{m}-1, b^{n}-1\right)$ when $a$ and $b$ are polynomials over arbitrary fields of positive characteristic, along with other generalizations on the same theme. Finally, we mention the work of Denis [Den11] who studied the same problem of the greatest common divisor in the context of Drinfeld modules. As hinted in [Sil04b], this greatest common divisor (GCD) problem may be studied in much higher generality; for example, if one knew the result of DeMarco-Mavraki [DM] (see Theorem 2.3) in the context of abelian varieties, then our method would extend to a similar conclusion for arbitrary abelian schemes over a base curve.

Our Theorem 1.1 is related also to [BC16, Theorem 1.1] (see also the extension from $[\mathrm{BC}]$ ) where it is shown that given $n$ linearly independent sections $P_{i}$ on the Legendre elliptic family $y^{2}=x(x-1)(x-t)$, there are at most finitely many parameters $t$ such that the points $\left(P_{i}\right)_{t}$ satisfy two independent linear relations on the corresponding elliptic curve. Therefore, a special case of the result by Barroero and Capuano is that given sections $P_{1}, P_{2}, Q_{1}, Q_{2}$ on the Legendre elliptic surface, if these 4 sections are linearly independent, then there are at most finitely many $t$ such that for some 
$m_{t}, n_{t} \in \mathbb{Z}$ we have that $\left[m_{t}\right]\left(P_{1}\right)_{t}=\left(Q_{1}\right)_{t}$ and $\left[n_{t}\right]\left(P_{2}\right)_{t}=\left(Q_{2}\right)_{t}$. However, in our Theorem 1.1 we obtain the same conclusion under the weaker hypothesis that $Q_{i}$ is not a multiple of $P_{i}$ for $i=1,2$ and also that $P_{1}$ and $P_{2}$ are linearly independent.

We also note that a special case of our Theorem 1.1 bears a resemblance to the classical Mordell-Lang problem (see [Fal94]). Indeed, with the notation as in Theorem 1.1, assume there exist infinitely many $t \in C(\overline{\mathbb{Q}})$ such that for some $m_{t} \in \mathbb{Z}$ we have

$$
\left[m_{t}\right]\left(P_{i}\right)_{t}=\left(Q_{i}\right)_{t} \text { for } i=1,2 .
$$

Also assume there is no $m \in \mathbb{Z}$ such that $[m] P_{i}=Q_{i}$ for $i=1,2$. Then the conclusion of Theorem 1.1 yields the existence of isogenies $\varphi: E_{1} \longrightarrow E_{2}$ and $\psi: E_{2} \longrightarrow E_{2}$ such that $\varphi\left(P_{1}\right)=\psi\left(P_{2}\right)$. Thus, using that (1.2) holds for infinitely many $t \in C(\overline{\mathbb{Q}})$ we get that

$$
\varphi\left(Q_{1}\right)=\psi\left(Q_{2}\right) .
$$

Therefore, if we let $X \subset \mathcal{A}:=\mathcal{E}_{1} \times \mathcal{E}_{2}$ be the 1-dimensional subscheme corresponding to the section $\left(Q_{1}, Q_{2}\right)$, and we let $\Gamma \subset \mathcal{A}$ be the cyclic subgroup spanned by $\left(P_{1}, P_{2}\right)$, then the existence of infinitely many $\gamma \in \Gamma$ such that for some $t \in C(\overline{\mathbb{Q}})$ we have $\gamma_{t} \in X$ yields that $X$ is contained in a proper algebraic subgroup of $\mathcal{A}$ (as given by the equation (1.3)). Such a statement can be viewed as a relative version of the classical Mordell-Lang problem; note that if $\mathcal{E}_{1}$ and $\mathcal{E}_{2}$ are constant elliptic surfaces with generic fibers $E_{i}^{0}$ defined over $\overline{\mathbb{Q}}$, while $\Gamma \subset\left(E_{1}^{0} \times E_{2}^{0}\right)(\overline{\mathbb{Q}})$, then this question is a special case of Faltings' theorem [Fal94] (formerly known as the MordellLang conjecture). It is natural to ask whether the above relative version of the Mordell-Lang problem holds more generally when $\mathcal{A} \longrightarrow C$ is an arbitrary semiabelian scheme, $X \subset \mathcal{A}$ is a 1-dimensional scheme and $\Gamma \subset \mathcal{A}$ is an arbitrary finitely generated group. This more general question is also related to the bounded height problems studied in [AMZ] in the context of pencils of finitely generated subgroups of $\mathbb{G}_{m}^{n}$.

In the next section of this paper, we review some preliminary material. Following that, in Section 3, we prove Theorem 1.1. The proof in the case of nonconstant sections is quite similar to the proofs of the main results of [AR04] and [HT], while the case of constant sections requires a different argument. In Section 4, we give a positive answer to Silverman's conjecture [Sil04b, Conjecture 7].

Acknowledgments. We thank Myrto Mavraki and Joe Silverman for several useful conversations.

\section{Preliminaries}

From now on, we fix an elliptic surface $\pi: \mathcal{E} \longrightarrow C$, where $C$ is a projective, smooth curve defined over $\overline{\mathbb{Q}}$. We denote by $E$ the generic fiber of $\mathcal{E}$; this is an elliptic curve defined over $\overline{\mathbb{Q}}(C)$. For all but finitely many $t \in C(\overline{\mathbb{Q}})$, we have that $\mathcal{E}_{t}:=\pi^{-1}(\{t\})$ is an elliptic curve defined over $\overline{\mathbb{Q}}$. 
2.1. Isotriviality. We say that $\mathcal{E}$ is isotrivial if the $j$-invariant of the generic fiber is a constant function (on $C$ ); for isotrivial elliptic surfaces $\mathcal{E}$, all smooth fibers of $\pi$ are isomorphic (to the generic fiber $E$ ). If $\mathcal{E}$ is isotrivial, then there exists a finite cover $C^{\prime} \longrightarrow C$ such that $\mathcal{E}^{\prime}:=\mathcal{E} \times{ }_{C} C^{\prime}$ is a constant (elliptic) surface over $C^{\prime}$, i.e., there exists an elliptic curve $E^{0}$ defined over $\overline{\mathbb{Q}}$ such that $\mathcal{E}^{\prime}=E^{0} \times_{\operatorname{Spec}(\overline{\mathbb{Q}})} C^{\prime}$. Furthermore, for a constant elliptic surface $E^{0} \times_{\operatorname{Spec}(\overline{\mathbb{Q}})} C^{\prime}$, we say that $\sigma_{P}$ is a constant section if $P \in E^{0}(\overline{\mathbb{Q}})$.

2.2. Canonical height on an elliptic surface. For each $t \in C(\overline{\mathbb{Q}})$ such that $\mathcal{E}_{t}$ is an elliptic curve, we let $\widehat{h}_{\mathcal{E}_{t}}$ be the Néron-Tate canonical height for the points in $\mathcal{E}_{t}(\overline{\mathbb{Q}})$ (for more details, see [Sil86]). There are two important properties of the canonical height which we will use:

(1) $\widehat{h}_{\mathcal{E}_{t}}\left(P_{t}\right)=0$ if and only if $P_{t}$ is a torsion point of $\mathcal{E}_{t}$, i.e., there exists a positive integer $k$ such that $[k] P_{t}=0$.

(2) for each $k \in \mathbb{Z}$ we have that $\widehat{h}_{\mathcal{E}_{t}}\left([k] P_{t}\right)=k^{2} \cdot \widehat{h}_{\mathcal{E}_{t}}\left(P_{t}\right)$.

Also, we let $\widehat{h}_{E}$ be the Néron-Tate canonical height on the generic fiber $E$ constructed with respect to the Weil height on the function field $\overline{\mathbb{Q}}(C)$ (for more details, see [Sil94a]). Property (2) above holds also on the generic fiber, i.e., $\widehat{h}_{E}([k] P)=k^{2} \cdot \widehat{h}_{E}(P)$. On the other hand, property (1) above holds only if $\mathcal{E}$ is non-isotrivial. Furthermore, if $\mathcal{E}=E \times{ }_{C} C$ is a constant family (where $E$ is an elliptic curve defined over $\overline{\mathbb{Q}}$ ), then for any $P \in E(\overline{\mathbb{Q}}(C)$ ), we have that $\widehat{h}_{E}(P)=0$ if and only if $P \in E(\overline{\mathbb{Q}})$.

2.3. Variation of the canonical height. We let $h_{C}$ be a given Weil height for the points in $C(\overline{\mathbb{Q}})$ corresponding to a divisor of degree 1 on $C$.

Let $P$ be a section of the elliptic surface $\mathcal{E} \longrightarrow C$. As before, we identify this section with its intersection (which we also call $P$ ) with the generic fiber of $\mathcal{E}$; also, for all but finitely many $t \in C(\overline{\mathbb{Q}})$, we have that the intersection of the image of $\sigma_{P}$ in $\mathcal{E}$ with the fiber above $t$ is a point $P_{t}$ on the elliptic curve $\mathcal{E}_{t}$. The following important fact will be used in our proof (see [CS93]):

$$
\lim _{h_{C}(t) \rightarrow \infty} \frac{\widehat{h}_{\mathcal{E}_{t}}\left(P_{t}\right)}{h_{C}(t)}=\widehat{h}_{E}(P) .
$$

Furthermore, the following more precise result holds, as proven by Silverman [Sil94b],

$$
\widehat{h}_{\mathcal{E}_{t}}\left(P_{t}\right)=h_{C, \eta(P)}(t)+O_{P}(1),
$$

where $\eta(P)$ is a divisor on $C$ of degree equal to $\widehat{h}_{E}(P)$ and $h_{C, \eta(P)}$ is a given Weil height for the points in $C(\overline{\mathbb{Q}})$ corresponding to the divisor $\eta(P)$, while the implicit constant from the term $O_{P}(1)$ is only dependent on the section $P$, but not on $t \in C(\overline{\mathbb{Q}})$. 
2.4. Points of small height on sections. We will use the following result of DeMarco-Mavraki [DM, Theorem 1.4] who extends [DWY16] (and in turn, uses the extensive analysis from [Sil94b] regarding the variation of the canonical height in an elliptic fibration). We also note that the case of isotrivial elliptic curves from Theorem 2.3 was previously proven by Zhang [Zha98], as part of Zhang's famous proof of the classical Bogomolov conjecture.

Theorem 2.3 (DeMarco-Mavraki [DM]). Let $\mathcal{E}_{1}, \mathcal{E}_{2}$ be elliptic fibrations over the same $\overline{\mathbb{Q}}$-curve $C$. Let $P_{i}$ be a section of $\mathcal{E}_{i}$ (for $i=1,2$ ) with the property that there exists an infinite sequence $\left\{t_{n}\right\} \subset C(\overline{\mathbb{Q}})$ such that

$$
\lim _{n \rightarrow \infty} \widehat{h}_{\left(\mathcal{E}_{i}\right)_{t_{n}}}\left(\left(P_{i}\right)_{t_{n}}\right)=0 \text { for } i=1,2
$$

Then there exist group homomorphisms $\phi: \mathcal{E}_{1} \longrightarrow \mathcal{E}_{2}$ and $\psi: \mathcal{E}_{2} \longrightarrow \mathcal{E}_{2}$, not both trivial, such that $\phi\left(P_{1}\right)=\psi\left(P_{2}\right)$.

\section{Proof of our main Result}

Propositions 3.1 and 3.9 are key to our proof.

Proposition 3.1. Let $C$ be a projective, smooth curve defined over $\overline{\mathbb{Q}}$, and let $h_{C}(\cdot)$ be a Weil height for the algebraic points of $C$ corresponding to a divisor of degree 1 . Let $P$ and $Q$ be sections of an elliptic surface $\pi: \mathcal{E} \longrightarrow C$ with generic fiber $E$, and assume there exists no $k \in \mathbb{Z}$ such that $[k] P=$ $Q$. In addition, assume $\widehat{h}_{E}(P)>0$. If there exists an infinite sequence $\left\{t_{i}\right\} \subset C(\overline{\mathbb{Q}})$ such that for each $i \in \mathbb{N}$ there exists some $m_{i} \in \mathbb{Z}$ such that $\left[m_{i}\right] P_{t_{i}}=Q_{t_{i}}$, then $h_{C}\left(t_{i}\right)$ is uniformly bounded and $\lim _{i \rightarrow \infty} \widehat{h}_{\mathcal{E}_{t_{i}}}\left(P_{t_{i}}\right)=0$.

Proof. Since $\left[m_{i}\right] P_{t_{i}}=Q_{t_{i}}$, we get that

$$
m_{i}^{2} \cdot \widehat{h}_{\mathcal{E}_{t_{i}}}\left(P_{t_{i}}\right)=\widehat{h}_{\mathcal{E}_{t_{i}}}\left(Q_{t_{i}}\right) .
$$

Since $[k] P \neq Q$ for any $k \in \mathbb{Z}$ and the sequence $\left\{t_{i}\right\}$ is infinite, then

$$
\lim _{i \rightarrow \infty}\left|m_{i}\right|=\infty \text {. }
$$

We claim first that $h_{C}\left(t_{i}\right)$ is uniformly bounded. Indeed, assuming (at the expense, perhaps, of replacing $\left\{t_{i}\right\}$ by an infinite subsequence) that $\lim _{i \rightarrow \infty} h_{C}\left(t_{i}\right)=\infty$, equation (2.1) coupled with equations (3.2) and (3.3) yields a contradiction. To see this, we divide both sides of $(3.2)$ by $h_{C}\left(t_{i}\right)$ and then take limits. Because $\widehat{h}_{E}(P)>0$, equation (3.3) yields that the left hand side equals

$$
\lim _{i \rightarrow \infty} m_{i}^{2} \cdot \frac{\widehat{h}_{\mathcal{E}_{i}}\left(P_{t_{i}}\right)}{h_{C}\left(t_{i}\right)}=\infty,
$$

while the right hand side equals

$$
\lim _{i \rightarrow \infty} \frac{\widehat{h}_{\mathcal{E}_{t_{i}}}\left(Q_{t_{i}}\right)}{h_{C}\left(t_{i}\right)}=\widehat{h}_{E}(Q)<\infty,
$$


which is a contradiction. So, indeed, we must have that $h_{C}\left(t_{i}\right)$ is uniformly bounded.

Next we prove that also $\widehat{h}_{\mathcal{E}_{t_{i}}}\left(Q_{t_{i}}\right)$ is uniformly bounded. Using (2.2) (see [Sil94b]), we know that there exists a divisor $\eta(Q)$ of $C$ of degree equal to $\widehat{h}_{E}(Q)$ such that

$$
\widehat{h}_{\mathcal{E}_{t}}\left(Q_{t}\right)=h_{C, \eta(Q)}(t)+O(1),
$$

where $h_{C, \eta(Q)}$ is a Weil height on $C(\overline{\mathbb{Q}})$ corresponding to the divisor $\eta(Q)$. Since $h_{C}$ is a Weil height associated to a divisor $D$ on $C$ of degree 1 , then for any positive integer $m>\operatorname{deg}(\eta(Q))$, the divisor $D_{1}:=m D-\eta(Q)$ has positive degree and therefore, it is ample. Then [HS00, Proposition B.3.2] yields that any Weil height $h_{C, D_{1}}$ associated to the divisor $D_{1}$ satisfies $h_{C, D_{1}}(t) \geq O(1)$ for all $t \in C(\overline{\mathbb{Q}})$. So,

$$
m h_{C}(t)+O(1) \geq h_{C, \eta(Q)}(t) \text { for } t \in C(\overline{\mathbb{Q}}) .
$$

Therefore $h_{C, \eta(Q)}\left(t_{i}\right)$ is uniformly bounded (since $h_{C}\left(t_{i}\right)$ is uniformly bounded). Then equation (3.6) provides the desired claim that

$$
\widehat{h}_{\mathcal{E}_{t_{i}}}\left(Q_{t_{i}}\right) \text { is bounded as } i \rightarrow \infty \text {. }
$$

Combining equations (3.2), (3.3) and (3.8), we finish the proof of Proposition 3.1.

Proposition 3.9. Let $P$ and $Q$ be sections of a constant elliptic fibration $\pi: \mathcal{E} \longrightarrow C$, and assume there exists no $k \in \mathbb{Z}$ such that $[k] P=Q$. In addition, assume $P$ is a non-torsion, constant section. If there exists an infinite sequence $\left\{t_{i}\right\} \subset C(\overline{\mathbb{Q}})$ such that for each $i \in \mathbb{N}$ there exists some $m_{i} \in \mathbb{Z}$ such that $\left[m_{i}\right] P_{t_{i}}=Q_{t_{i}}$, then $\lim _{i \rightarrow \infty} h_{C}\left(t_{i}\right)=\infty$.

Proof. We have that each fiber $\mathcal{E}_{t_{i}}$ is isomorphic to the generic fiber $E^{0}$, and so, because $P$ is a constant section,

$$
\widehat{h}_{\mathcal{E}_{t_{i}}}\left(P_{t_{i}}\right)=\widehat{h}_{E^{0}}\left(P^{0}\right),
$$

where $P^{0}$ is the intersection of $P$ with the generic fiber and $\widehat{h}_{E^{0}}(\cdot)$ is the Néron-Tate canonical height of the elliptic curve $E^{0}$ defined over $\overline{\mathbb{Q}}$ (i.e., it is not the canonical height on the generic fiber of $\mathcal{E}$ seen as an elliptic curve defined over the function field $\overline{\mathbb{Q}}(C)$ ).

Furthermore, since $P^{0}$ is not a torsion point of $E^{0}$, then $\widehat{h}_{E^{0}}\left(P^{0}\right)>0$. Thus, from the equality $\left[m_{i}\right] P_{t_{i}}=Q_{t_{i}}$, along with equation (3.10) coupled with the fact that $\left|m_{i}\right| \rightarrow \infty$ (because $[k] P \neq Q$ for all integers $k$ ), we get that

$$
\widehat{h}_{\mathcal{E}_{t_{i}}}\left(Q_{t_{i}}\right)=m_{i}^{2} \widehat{h}_{E^{0}}\left(P^{0}\right) \rightarrow \infty .
$$

Then, using (2.2), we have

$$
\widehat{h}_{\mathcal{E}_{t_{i}}}\left(Q_{t_{i}}\right)=h_{C, \eta(Q)}\left(t_{i}\right)+O(1)
$$


where $h_{C, \eta(Q)}$ is a Weil height on $C$ corresponding to a divisor $\eta(Q)$. So, equations (3.11) and (3.12) yield $h_{C, \eta(Q)}\left(t_{i}\right) \rightarrow \infty$ and thus, $h_{C}\left(t_{i}\right) \rightarrow \infty$ (see [HS00, Proposition B.3.5], along with our similar argument from the proof of Proposition 3.1).

Now we can prove our main result.

Proof of Theorem 1.1. First we note that if $P_{i}$ is a torsion section (for some $i \in\{1,2\}$ ), then conclusion (ii) holds trivially since then we would get that there exist infinitely many $t \in C(\overline{\mathbb{Q}})$ such that $\left(Q_{i}\right)_{t}=[k]\left(P_{i}\right)_{t}$ for the same integer $k$. So, from now on, we assume that both $P_{1}$ and $P_{2}$ are nontorsion sections on $\mathcal{E}_{1}$, respectively $\mathcal{E}_{2}$. In particular, this means that if $\widehat{h}_{E_{i}}\left(P_{i}\right)=0$, then $\mathcal{E}_{i}$ must be an isotrivial elliptic surface.

We assume there exists an infinite sequence $\left\{t_{i}\right\} \subset C(\overline{\mathbb{Q}})$ such that for each $i \in \mathbb{N}$ there exist $m_{i, 1}, m_{i, 2} \in \mathbb{Z}$ with the property that $\left[m_{i, 1}\right]\left(P_{1}\right)_{t_{i}}=\left(Q_{1}\right)_{t_{i}}$ and also $\left[m_{i, 2}\right]\left(P_{2}\right)_{t_{i}}=\left(Q_{2}\right)_{t_{i}}$. In addition, we assume conclusion (ii) does not hold, i.e., there is no $m \in \mathbb{Z}$ such that $[m] P_{i}=Q_{i}$ for some $i \in\{1,2\}$. We split our analysis into two cases.

Case 1. $\widehat{h}_{E_{i}}\left(P_{i}\right)>0$ for each $i=1,2$.

Applying then Proposition 3.1 to the sections $P_{i}$ and $Q_{i}$, we get that

$$
\lim _{i \rightarrow \infty} \widehat{h}_{\left(\mathcal{E}_{1}\right)_{t_{i}}}\left(\left(P_{1}\right)_{t_{i}}\right)=\lim _{i \rightarrow \infty} \widehat{h}_{\left(\mathcal{E}_{2}\right)_{t_{i}}}\left(\left(P_{2}\right)_{t_{i}}\right)=0 .
$$

Equation (3.13) along with Theorem 2.3 yields that conclusion (i) must hold in Theorem 1.1. Note that we obtain in this case that the morphisms $\varphi: E_{1} \longrightarrow E_{2}$ and $\psi: E_{2} \longrightarrow E_{2}$ from the conclusion of Theorem 2.3 are both isogenies since $P_{1}$ and $P_{2}$ are nontorsion sections.

Case 2. Either $\widehat{h}_{E_{1}}\left(P_{1}\right)=0$ or $\widehat{h}_{E_{2}}\left(P_{2}\right)=0$.

Without loss of generality, we assume $\widehat{h}_{E_{1}}\left(P_{1}\right)=0$. Therefore (since $P_{1}$ is not torsion) $\mathcal{E}_{1}$ is an isotrivial elliptic surface, and furthermore, at the expense of replacing $C$ by a finite cover (and also base extending $\mathcal{E}_{1}$ and respectively $\mathcal{E}_{2}$ ), we may assume that $\mathcal{E}_{1}$ is a constant family. Thus, $\mathcal{E}_{1}=E_{1}^{0} \times{ }_{C} C$ for some elliptic curve $E_{1}^{0}$ defined over $\overline{\mathbb{Q}}$. Then also $P$ is a constant (nontorsion) section, because $\widehat{h}_{\mathcal{E}_{1}}\left(P_{1}\right)=0$. Finally, we let $h_{C}(\cdot)$ be a Weil height for the algebraic points of $C$ with respect to a divisor of degree 1.

If $\widehat{h}_{E_{2}}\left(P_{2}\right)>0$, then Proposition 3.1 applied to $P_{2}$ and $Q_{2}$ yields that $h_{C}\left(t_{i}\right)$ is uniformly bounded, which contradicts the conclusion of Proposition 3.9 applied to $P_{1}$ and $Q_{1}$. Therefore, we must have that $\widehat{h}_{E_{2}}\left(P_{2}\right)=0$ and therefore, also $\mathcal{E}_{2}$ is an isotrivial elliptic surface. At the expense of (yet another) base extension, we may assume that also $\mathcal{E}_{2}=E_{2}^{0} \times C$ is a constant fibration. Then $P_{2}$ is a constant, nontorsion section on $\mathcal{E}_{2}$. We let $P_{i}^{0}$ be the intersection of $P_{i}$ (for $i=1,2$ ) with the generic fiber of $\mathcal{E}_{i}$.

Now, if either $Q_{1}$ or $Q_{2}$ is also a constant section, then we get a contradiction since we assumed conclusion (ii) does not hold. Indeed, if for some $i=1,2$ we have that both $P_{i}$ and $Q_{i}$ are constant sections on the 
constant elliptic surface $\mathcal{E}_{i}$, then the existence of a point $t \in C(\overline{\mathbb{Q}})$ such that for some $k \in \mathbb{Z}$ we have $[k]\left(P_{i}\right)_{t}=\left(Q_{i}\right)_{t}$ yields that actually $[k] P_{i}=Q_{i}$ on $\mathcal{E}_{i}$. So, we may assume that $Q_{1}$ and $Q_{2}$ are both nonconstant sections on $\mathcal{E}_{1}$, respectively $\mathcal{E}_{2}$. Then there is a (neither vertical, nor horizontal) curve $X \subset E_{1}^{0} \times E_{2}^{0}$ containing all points $\left(\left(Q_{1}\right)_{t},\left(Q_{2}\right)_{t}\right)$ for $t \in C(\overline{\mathbb{Q}})$. Furthermore, our hypothesis yields that this curve $X$ intersects the subgroup $\Gamma \subset E_{1}^{0} \times E_{2}^{0}$ spanned by the points $\left(P_{1}^{0}, 0\right)$ and $\left(0, P_{2}^{0}\right)$ in an infinite set. Then the classical Mordell-Lang conjecture (proven by Faltings [Fa194]) yields that $X$ itself is a coset of an algebraic subgroup of $E_{1}^{0} \times E_{2}^{0}$. Hence, because $X$ projects dominantly onto each coordinate, there exists a nontrivial isogeny $\tau: E_{1}^{0} \longrightarrow E_{2}^{0}$, and also there exist endomorphisms $\phi_{i}$ of $E_{i}^{0}$, not both trivial, such that $\tau\left(\phi_{1}\left(Q_{1}\right)\right)=\phi_{2}\left(Q_{2}\right)$. Then, using (for any $i$ such that $m_{i, 1}$ and $m_{i, 2}$ are nonzero) that

$$
\left[m_{i, 1}\right] P_{1}^{0}=\left(Q_{1}\right)_{t_{i}} \text { and }\left[m_{i, 2}\right] P_{2}^{0}=\left(Q_{2}\right)_{t_{i}}
$$

along with the fact that $\tau\left(\phi_{1}\left(\left(Q_{1}\right)_{t_{i}}\right)\right)=\phi_{2}\left(\left(Q_{2}\right)_{t_{i}}\right)$, we obtain the conclusion in Theorem 1.1 with $\varphi:=\tau \circ\left[m_{i, 1}\right] \circ \phi_{1}$ and $\psi:=\left[m_{i, 2}\right] \circ \phi_{2}$. Finally, note that since $P_{1}$ and $P_{2}$ are non-torsion, then also $\varphi$ and $\psi$ are dominant morphisms.

\section{Common divisors of Elliptic Sequences}

In this section, we apply Theorem 1.1 to prove Silverman's conjecture [Sil04b, Conjecture 7] concerning common divisors of elliptic sequences; actually, our Proposition 4.3 provides a slightly more general answer than [Sil04b, Conjecture 7]. We first recall the terminology and notation from [Sil04b] that we will use in this section.

Let $k$ be an algebraically closed field of characteristic 0 . Let $C$ be a smooth projective curve defined over $k$ and let $K=k(C)$ be the function field of $C$. For any point $\gamma \in C(k)$, we let $\operatorname{ord}_{\gamma}(D)$ denote the coefficient of $\gamma$ in $D \in \operatorname{Div}(C)$. The greatest common divisor for any two effective divisors $D_{1}, D_{2} \in \operatorname{Div}(C)$ is defined as

$$
\operatorname{GCD}\left(D_{1}, D_{2}\right)=\sum_{\gamma \in C} \min \left\{\operatorname{ord}_{\gamma}\left(D_{1}\right), \operatorname{ord}_{\gamma}\left(D_{2}\right)\right\} \cdot(\gamma) \in \operatorname{Div}(C)
$$

For an elliptic curve $E$ defined over $K$, let $\pi: \mathcal{E} \longrightarrow C$ be an elliptic surface whose generic fiber is $E$ and let $P \in E(K)$. Recall that the section corresponding to $P$ is denoted by $\sigma_{P}: C \rightarrow \mathcal{E}$. We denote the image of $\sigma_{P}$ by $\bar{P}:=\sigma_{P}(C) \subset \mathcal{E}$.

Let $E_{1}$ and $E_{2}$ be elliptic curves defined over $K$, let $\mathcal{E}_{i} / C$ be elliptic surfaces with generic fibers $E_{i}$, and let $P_{i} \in E_{i}(K)$ for $i=1,2$. The greatest common divisor of $P_{1}$ and $P_{2}$ is given by

$$
\operatorname{GCD}\left(P_{1}, P_{2}\right)=\operatorname{GCD}\left(\sigma_{P_{1}}^{*}\left(\bar{O}_{\mathcal{E}_{1}}\right), \sigma_{P_{2}}^{*}\left(\bar{O}_{\mathcal{E}_{2}}\right)\right) .
$$

where $\bar{O}_{\mathcal{E}_{i}}:=\sigma_{O_{i}}(C)$ is the zero section on $\mathcal{E}_{i}$ corresponding to the identity $O_{i}$ of $E_{i}, i=1,2$. Thus, for any given $Q_{i} \in E_{i}(K), \operatorname{GCD}\left(P_{1}-Q_{1}, P_{2}-Q_{2}\right)$ 
is the greatest common divisor of the two points $P_{i}-Q_{i} \in E_{i}$ for $i=1,2$. In the following, points $P_{1}$ and $P_{2}$ are called $(K-)$ dependent if there are morphisms $\varphi: E_{1} \longrightarrow E_{2}$ and $\psi: E_{2} \longrightarrow E_{2}$ not both trivial such that $\varphi\left(P_{1}\right)=\psi\left(P_{2}\right)$; otherwise they are called independent. Note that if one of $P_{1}$ and $P_{2}$ is a torsion point, then they are automatically dependent.

Motivated by Ailon-Rudnick's result [AR04], Silverman conjectured that an elliptic analogue also exists. For the convenience of the reader, we recall his conjecture as follows.

Conjecture 4.1 (Silverman [Sil94b, Conjecture 7]). Let $K=k(C)$ be the function field of a smooth projective curve $C$ over an algebraically closed field $k$ of characteristic 0 , let $E_{1} / K$ and $E_{2} / K$ be elliptic curves, and let $P_{1} \in E_{1}(K)$ and $P_{2} \in E_{2}(K)$ be $K$-independent points.

(i) There is a constant $c=c\left(K, E_{1}, E_{2}, P_{1}, P_{2}\right)$ so that

$$
\operatorname{deg} \operatorname{GCD}\left(\left[n_{1}\right] P_{1},\left[n_{2}\right] P_{2}\right) \leq c \text { for all } n_{1}, n_{2} \geq 1 \text {. }
$$

(ii) Further, there is an equality

$$
\operatorname{GCD}\left([n] P_{1},[n] P_{2}\right)=\operatorname{GCD}\left(P_{1}, P_{2}\right) \quad \text { for infinitely many } n \geq 1 \text {. }
$$

Remark 4.2. Silverman [Sil94b, Theorem 8] showed that Conjecture 4.1 is true provided that both $E_{1}$ and $E_{2}$ have constant $j$-invariant.

As an application of Theorem 1.1, we prove that Conjecture 4.1 holds (even in a slightly stronger form); we strengthen further the conclusion from Conjecture 4.1 when $k=\overline{\mathbb{Q}}$.

Proposition 4.3. Let $k$ be an algebraically closed field of characteristic 0 . Let $C$ be a smooth projective curve defined over $k$, let $K=k(C)$ and let $E_{i} / K, i=1,2$, be elliptic curves defined over $K$. Let $P_{i}, Q_{i} \in E_{i}(K)$ for $i=1,2$ and furthermore, assume that $P_{1}$ and $P_{2}$ are $K$-independent.

(i) If $k=\overline{\mathbb{Q}}$, then there exists an effective divisor $D \in \operatorname{Div}(C)$ such that

$$
\operatorname{GCD}\left(\left[n_{1}\right] P_{1}-Q_{1},\left[n_{2}\right] P_{2}-Q_{2}\right) \leq D
$$

for all integers $n_{i}$ such that $\left[n_{i}\right] P_{i} \neq Q_{i}, i=1,2$.

(ii) For an arbitrary algebraically closed field $k$ of characteristic 0 , there exists an effective divisor $D_{0} \in \operatorname{Div}(C)$ such that

$$
\operatorname{GCD}\left(\left[n_{1}\right] P_{1},\left[n_{2}\right] P_{2}\right) \leq D_{0}
$$

for all nonzero integers $n_{i}$.

(iii) The set

$$
\left\{n \geq 1: \operatorname{GCD}\left([n] P_{1},[n] P_{2}\right)=\operatorname{GCD}\left(P_{1}, P_{2}\right)\right\}
$$

has positive density in $\mathbb{N}$.

(iv) For all but finitely many primes $q$, we have that $\operatorname{GCD}\left([q] P_{1},[q] P_{2}\right)=$ $\operatorname{GCD}\left(P_{1}, P_{2}\right)$. 
Remark 4.4. The conclusion of Proposition 4.3 (i) for an arbitrary algebraically closed field $k$ of characteristic 0 would follow from our method once the validity of DeMarco-Mavraki's result [DM] (see Theorem 2.3) is extended over function fields. In turn, their result is contingent on establishing the smooth variation of the canonical height in fibers of an elliptic surface defined over a function field (over $\overline{\mathbb{Q}}$ ).

The proof of Proposition 4.3 relies on Theorem 1.1 and the following lemma which is a variant of [Sil04b, Lemma 4] bounding $\operatorname{ord}_{\gamma}\left(\sigma_{[n] P}^{*}\left(\bar{O}_{\mathcal{E}}\right)\right)$ for $\gamma \in C$ and all integers $n \neq 0$.

Lemma 4.5. Let $k$ be an algebraically closed field of characteristic 0. Let $E$ be an elliptic curve defined over $k$ and let $\mathcal{E} \longrightarrow C$ be an elliptic surface whose generic fiber is $E$. Let $\gamma \in C(k)$ and let $P, Q \in E(k(C))$ be given. There exists a constant $m=m(\gamma, E, P, Q)$ such that $\operatorname{ord}_{\gamma}\left(\sigma_{[n] P}^{*}(\bar{Q})\right) \leq m$ for all integers $n$ such that $[n] P \neq Q$.

Proof. Observe that $\operatorname{ord}_{\gamma}\left(\sigma_{[n] P}^{*}(\bar{Q})\right) \geq 1$ if and only if $\sigma_{[n] P}(\gamma)=\sigma_{Q}(\gamma)$. Moreover, $\sigma_{Q}(\gamma)$ is a torsion point of $\mathcal{E}_{\gamma}$ if and only if there are more than one $n$ such that $\operatorname{ord}_{\gamma}\left(\sigma_{[n] P}^{*}(\bar{Q})\right) \geq 1$.

It suffices to prove the assertion when $\operatorname{ord}_{\gamma}\left(\sigma_{n P}^{*}(\bar{Q})\right) \geq 1$ for more than one integer $n$. Thus, we assume that $\sigma_{Q}(\gamma)$ is a torsion point of $\mathcal{E}_{\gamma}$. Let $\ell$ be the order of $\sigma_{Q}(\gamma)$ and that $\operatorname{ord}_{\gamma}\left(\sigma_{n P}^{*}(\bar{Q})\right) \geq 1$ for some integer $n$ such that $[n] P \neq Q$. It follows that $\operatorname{ord}_{\gamma}\left(\sigma_{n P}^{*}(\bar{Q})\right)$ is finite and that

$$
\sigma_{[\ell n] P}(\gamma)=[\ell] \sigma_{[n] P}(\gamma)=[\ell] \sigma_{Q}(\gamma)=O_{\mathcal{E}_{\gamma}},
$$

which is the zero element for the elliptic curve $\mathcal{E}_{\gamma}$.

If $Q$ is the zero element of $E$, then it follows from [Sil04b, Lemma 4] that the value of $\operatorname{ord}_{\gamma}\left(\sigma_{[n] P}^{*}\left(\bar{O}_{\mathcal{E}}\right)\right)$ is bounded independent of $n \neq 0$ and we are done in this case.

Assume that $Q \neq O$. Then (4.6) yields the inequality

$$
\operatorname{ord}_{\gamma}\left(\sigma_{[n] P}^{*}(\bar{Q})\right) \leq \operatorname{ord}_{\gamma}\left(\sigma_{[\ell n] P}^{*}\left(\bar{O}_{\mathcal{E}}\right)\right) .
$$

Hence, we know that $\operatorname{ord}_{\gamma}\left(\sigma_{[n] P}^{*}(\bar{Q})\right)$ is bounded independent of $n \neq 0$ (and $n$ such that $[n] P \neq Q)$. As $Q \neq O$, we also have that $\operatorname{ord}_{\gamma}\left(\sigma_{n P}^{*}(\bar{Q})\right)$ is finite if $n=0$. Thus we obtain that $\operatorname{ord}_{\gamma}\left(\sigma_{n P}^{*}(\bar{Q})\right)$ is bounded independent of $n$ such that $[n] P \neq Q$, which concludes our proof.

Proof of Proposition 4.3. We first prove part (i) in Proposition 4.3. So, for each $\gamma \in C(\overline{\mathbb{Q}})$, let $m_{i, \gamma}$ be an upper bound for $\operatorname{ord}_{\gamma}\left(\sigma_{[n] P_{i}}^{*}\left(\bar{Q}_{i}\right)\right)$ as in Lemma 4.5. Set $m_{\gamma}=\min \left\{m_{1, \gamma}, m_{2, \gamma}\right\}$. Since $P_{1}$ and $P_{2}$ are independent, by Theorem 1.1 we may take $m_{\gamma}=0$ for all but finitely many points $\gamma \in C(\overline{\mathbb{Q}})$. Put

$$
D:=\sum_{\gamma \in C(\overline{\mathbb{Q}})} m_{\gamma}(\gamma)
$$


Then, $D$ is an effective divisor of $C$. Now it follows directly from Lemma 4.5 that $\operatorname{GCD}\left(\left[n_{1}\right] P_{1}-Q_{1},\left[n_{2}\right] P_{2}-Q_{2}\right) \leq D$ for all $n_{i}$ such that $\left[n_{i}\right] P \neq Q_{i}$ for both $i=1,2$.

For the proof of part (ii) in Proposition 4.3, we let $Q_{i}=O_{i}$ be the zero element of $E_{i}$ for $i=1,2$. If $k=\overline{\mathbb{Q}}$, then the result follows immediately from part (i). Now, for the general case, we note that it suffices to prove the existence of at most finitely many $t \in C(k)$ such that both $\left(P_{1}\right)_{t}$ and $\left(P_{2}\right)_{t}$ are torsion points on the elliptic fiber $\mathcal{E}_{t}$; indeed, the fact that the multiplicity of each such $t$ appearing in a divisor $\operatorname{GCD}\left(\left[n_{1}\right] P_{1},\left[n_{2}\right] P_{2}\right)$ is bounded follows exactly as in the proof of part (i), using Lemma 4.5. On the other hand, if there exist infinitely many $t \in C(k)$ such that both $\left(P_{1}\right)_{t}$ and $\left(P_{2}\right)_{t}$ are torsion yields (according to [MZ14, Theorem, p. 117]) that $P_{1}$ and $P_{2}$ are related, contradiction.

The conclusion in part (iii) in Proposition 4.3 follows almost verbatim from the proof of [Sil04b, Theorem 8 (b)]. Essentially, the argument is as follows. For each of the finitely many $\gamma \in C(k)$ which does not appear in the support of $\operatorname{GCD}\left(P_{1}, P_{2}\right)$, but for which there exists some positive integer $n$ such that $\gamma$ is contained in the support of the divisor $\operatorname{GCD}\left([n] P_{1},[n] P_{2}\right)$, or equivalently,

$$
\text { the divisor } \operatorname{GCD}\left([n] P_{1},[n] P_{2}\right)-(\gamma) \text { is effective, }
$$

we let $n_{\gamma}$ be the smallest such positive integer $n$ for which (4.7) holds. Then it is easy to see that $\gamma$ is in the support of $\operatorname{GCD}\left([n] P_{1},[n] P_{2}\right)$ if and only if $n_{\gamma} \mid n$. Also, for each of these points $\gamma$ which are not in the support of $\operatorname{GCD}\left(P_{1}, P_{2}\right)$, we have that $n_{\gamma}>1$. Then for any positive integer $n$ which is not divisible by any of the finitely many integers $n_{\gamma}$, we have that

$$
\operatorname{GCD}\left([n] P_{1},[n] P_{2}\right)=\operatorname{GCD}\left(P_{1}, P_{2}\right) \text {. }
$$

The conclusion in part (iv) in Proposition 4.3 follows from the proof of part (iii) since for all primes $q$ which do not divide any of the finitely many numbers $n_{\gamma}>1$, we have that $\operatorname{GCD}\left([q] P_{1},[q] P_{2}\right)=\operatorname{GCD}\left(P_{1}, P_{2}\right)$.

\section{REFERENCES}

[AR04] N. Ailon and Z. Rudnick, Torsion points on curves and common divisors of $a^{k}-1$ and $b^{k}-1$, Acta Arith. 113 (2004), no. 1, 31-38.

[AMZ] F. Amoroso, D. Masser, and U. Zannier, Bounded height in pencils of finitely generated groups, preprint, arxiv 1509.04963.

[BC16] F. Barroero and L. Capuano, Linear relations in families of powers of elliptic curves, Algebra \& Number Theory 10 (2016), 195-214.

[BC] F. Barroero and L. Capuano, Unlikely intersections in families of products of elliptic curves and the multiplicative group, arxiv 1606.02063.

[BG06] E. Bombieri and W. Gubler, Heights in Diophantine geometry, New Mathematical Monographs, vol. 4, Cambridge University Press, Cambridge, 2006.

[BCZ03] Y. Bugeaud, P. Corvaja and U. Zannier, An upper bound for the G.C.D. of $a^{n}-1$ and $b^{n}-1$, Math. Z. 243 (2003), 79-84.

[CS93] G. S. Call and J. Silverman, Canonical heights on varieties with morphism, Compositio Math. 89 (1993), 163-205. 
[CZ08] P. Corvaja and U. Zannier, Some cases of Vojta's conjecture on integral points over function fields, J. Algebraic Geom. 17 (2008), 295-333; Addendum, Asian J. Math. 14 (2010), 581-584.

[CZ11] P. Corvaja and U. Zannier, An abcd theorem over function fields and applications, Bull. Soc. Math. France 139 (2011), 437-454.

[CZ13a] P. Corvaja and U. Zannier, Algebraic hyperbolicity of ramified covers of $\mathbb{G}_{m}^{2}$ (and integral points on affine subsets of $\mathbb{P}^{2}$ ), J. Differential Geom. 93 (2013), 355-377.

[CZ13b] P. Corvaja and U. Zannier, Greatest common divisors of $u-1, v-1$ in positive characteristic and rational points on curves over finite fields, J. Eur. Math. Soc. (JEMS) 15 (2013), no. 5, 1927-1942.

[DM] L. DeMarco and N. M. Mavraki, Variation of canonical height and equidistribution, preprint, arxiv 1701.07947v1.

[DWY16] L. DeMarco, X. Wang, and H. Ye, Torsion points and the Lattés family, Amer. J. Math. 138 (2016), no. 3, 697-732.

[Den11] L. Denis, Facteurs communs et torsion en caractéristique non nulle, J. Théor. Nombres Bordeaux 23 (2011), no. 2, 347-352.

[Fal94] G. Faltings, The general case of S. Lang's conjecture, Barsotti Symposium in Algebraic Geometry (Abano Terme, 1991), 175-182, Perspect. Math. 15, Academic Press, San Diego, CA, 1994.

[GHT17] D. Ghioca, L.-C. Hsia, and T. J. Tucker, On a variant of the Ailon-Rudnick theorem in finite characteristic, New York J. Math. 23 (2017), 213-225.

[HT] L.-C. Hsia and T. J. Tucker, Greatest common divisors of iterates of polynomials, preprint, arxiv 1611.04115.

[HS00] M. Hindry and J. H. Silverman, Diophantine geometry. An introduction, Graduate Texts in Mathematics 201, Springer-Verlag, New York, 2000, xiv +558 pp.

[Lan65] S. Lang, Division points on curves, Ann. Mat. Pura Appl. (4) 70 (1965), 229234.

[MZ14] D. Masser and U. Zannier, Torsion points on families of products of elliptic curves, Adv. Math. 259 (2014), 116-133.

[Sil83] J. H. Silverman, Heights and specialization map for families of abelian varieties, J. Reine Angew. Math. 342 (1983), 197-211.

[Sil86] J. H. Silverman, The arithmetic of elliptic curves, Second edition, Graduate Texts in Mathematics 106, Springer-Verlag, New York, 1986, xii+400 pp.

[Sil94a] J. H. Silverman, Advanced topics in the arithmetic of elliptic curves, Graduate Texts in Mathematics 151, Springer-Verlag, New York, 1994, xiv+525 pp.

[Sil94b] J. H. Silverman, Variation of the canonical height on elliptic surfaces. III. Global boundedness properties, J. Number Theory 48 (1994), no. 3, 330-352.

[Sil04a] J. H. Silverman, Common divisors of $a^{n}-1$ and $b^{n}-1$ over function fields, New York J. Math. 10 (2004), 37-43.

[Sil04b] J. H. Silverman, Common divisors of elliptic divisibility sequences over function fields, Manuscripta Math. 114 (2004), no. 4, 431-446.

[Zha98] S. Zhang, Equidistribution of small points on abelian varieties, Ann. of Math. (2) 147 (1998), no. 1, 159-165. 
Dragos Ghioca, Department of Mathematics, University of British Columbia, VAncouver, BC V6T 1Z2, CAnada

E-mail address: dghioca@math.ubc.ca

Liang-Chung Hsia, Department of Mathematics, National Taiwan Normal University, TAIPEI, TAIWAN, ROC

E-mail address: hsia@math.ntnu.edu.tw

Thomas Tucker, Department of Mathematics, University of Rochester, Rochester, NY 14627, USA

E-mail address: ttucker@math.rochester.edu 